\title{
Food Volume Effects on Intake and Appetite in Women with Binge-Eating Disorder and Weight-Matched Controls
}

Janet D. Latner, PhD ${ }^{1 *}$

Juliet K. Rosewall, BA (Hons) ${ }^{2}$

Amy M. Chisholm, BSc (Hons) ${ }^{2}$

\begin{abstract}
Objective: Our study examined the effect of food volume on later intake and appetite ratings in 15 women with binge eating disorder (BED) and 15 healthy control women.
\end{abstract}

Method: On nonconsecutive days, lowervolume $(250 \mathrm{ml} / 171 \mathrm{kcal})$ and highervolume milk-based preloads $(500 \mathrm{ml} / 171$ kcal) were served in counterbalanced order. Thirty minutes later, appetite ratings and intake at a buffet-style testlunch were assessed.

Results: Higher-volume preloads decreased hunger, desire to eat, excitement about eating, desire for dessert, and loss of control over eating at test meals, relative to lower-volume preloads. Test-meal intake was greater among BED partici- pants than controls; intake did not differ by preload volume. BED participants' desire to eat, prospective consumption, excitement about eating, and desire for meals and desserts were higher than controls' ratings.

Conclusion: Higher-volume foods decreased hunger and related appetite ratings. Differences in food intake and appetite between BED and control participants provide further evidence for the validity of test meals to assess binge eating. () 2008 by Wiley Periodicals, Inc.

Keywords: binge-eating disorder; food volume; food intake; satiety; hunger; test meals; preloads

(Int J Eat Disord 2008; 00:000-000)

\section{Introduction}

Considering the rapid rise in the prevalence of obesity $^{1}$ and the increased recognition of eating disorders that involve excessive food consumption, research on dietary interventions that can reduce food intake and prevent overeating has become increasingly important. Several studies have shown that the volume of food consumed can influence hunger ratings and food intake. Holding energy constant, Rolls and colleagues ${ }^{2}$ demonstrated that increasing the volume of milk-based drinks can decrease the subsequent food intake of lean men. Although this effect may be partly mediated by sensory-specific satiety, ${ }^{3}$ it may not rely solely on sensory cues. A similar reduction in food intake was found in both lean and obese women when a load

\footnotetext{
Accepted 26 April 2008

Supported by U6550 from University of Canterbury.

*Correspondence to: Janet Latner, Department of Psychology,

University of Hawaii at Manoa, 2430 Campus Road, Honolulu, HI.

E-mail: jlatner@hawaii.edu

${ }^{1}$ Department of Psychology, University of Hawaii at Manoa,

2430 Campus Road, Honolulu, Hawaii

${ }^{2}$ Department of Psychology, University of Canterbury,

Christchurch, New Zealand

Published online in Wiley InterScience

(www.interscience.wiley.com). DOI: 10.1002/eat.20570

(C) 2008 Wiley Periodicals, Inc.
}

of greater volume was infused intragastrically, relative to an equicaloric, lower volume load. ${ }^{4}$ In lean women, energy intake was higher at a lunch following a casserole than following a diluted soup containing the same ingredients. ${ }^{5}$ Even increasing food volume by the incorporation of air decreased subsequent intake. ${ }^{6}$ The effect of these variables on food intake may be related to their influence on the organs that control intake. These results are consistent with research on energy density, which is usually inversely related to the volume of foods and directly related to energy intake. ${ }^{7-9}$

However, other researchers have found that increased food volume influences appetite ratings, but not subsequent food intake. Three studies with healthy men and women found lower hunger and higher fullness ratings after higher-volume (HV) soup relative to lower-volume (LV) soup, but no effect on subsequent food intake. ${ }^{10-12}$ Different experimental designs across food volume studies make their results difficult to compare. However, studies showing an effect on food intake used multiple-item test meals, while those showing only the effect on appetite ratings used single-item meals. Most of these studies have used lean, healthy samples.

Given the potential therapeutic value of this effect for individuals with eating disorders and obesity, it is important to resolve whether both appetite 
ratings and food intake can be influenced by food volume. Individuals with binge-eating disorder (BED) are characterized by at least twice weekly binge eating: episodes of excessive food intake accompanied by a loss of control over eating. ${ }^{13}$ These individuals have numerous disturbances in the development of satiety that may account for these episodes. ${ }^{14}$ In particular, obese individuals with BED have increased gastric capacity relative to obese individuals without binge eating. ${ }^{15}$ Gastric capacity is highly correlated with mean intake during binge episodes. ${ }^{16,17}$ These findings suggest that binge eating is related to gastric capacity, while other research suggests that weight is also related to the amount of food consumed during binge episodes. ${ }^{18}$ Enlarged gastric capacity may interfere with gastric distention responses and could delay the release of cholecystokinin after food intake, leading to less satiety. ${ }^{15,19}$ Therefore, individuals with BED might need to consume greater volumes of food to achieve sufficient gastric distention to produce satiety.

Previous studies on the effects of food volume on intake and appetite have excluded individuals with eating disorders. However, women with BED might especially benefit from foods that decrease appetite and hunger signals. Therefore, this study aimed to extend previous research by examining the effects of food volume on appetite ratings and food intake in women with BED, as well as in healthy, weightmatched control women. This investigation examined whether different volume levels of a milkbased preload would differentially affect appetite and food intake at a multi-item test meal in women with or without BED. Previous research has shown that HV preloads reduced hunger ratings and food intake relative to LV preloads in healthy women and men. ${ }^{2,4,5}$ Based on these past studies, it was hypothesized that compared to LV preloads, HV preloads would decrease self-reported hunger and related appetite ratings and decrease food intake in women with BED and weight-matched controls.

\section{Method}

\section{Participants}

Posted flyers advertised for either women with regular overeating or healthy women to participate in research on eating patterns. Telephone screening assessed exclusionary criteria: substance use, physical conditions that influence appetite (including pregnancy, diabetes, or other metabolic conditions), serious mental or medical illness, or lactation. Women who reported binge eating during telephone screening were invited for an interview. Potential participants were interviewed by trained assessors using the Eating Disorder Examination $\left(\mathrm{EDE}^{; 0}{ }^{20}\right.$ ), a reliable and widely used instrument for the diagnosis of eating disorders. ${ }^{21}$ They were administered the Eating Attitudes Test $\left(\mathrm{EAT}^{2}{ }^{22}\right)$ a 26 -item screening measure that detects general eating disturbance, and the Depression, Anxiety, and Stress Scales (DASS; ${ }^{23}$ ), a 42 -item measure of depression, anxiety, and perceived stress. Height and weight were measured using a digital scale. Dislike for or allergies to any of the foods served in the study were assessed. Those who met Diagnostic and Statistical Manual of Mental Disorders (DSM-IV; ${ }^{24}$ ) criteria for BED were invited to participate.

Weight-matched controls were recruited following BED participants so that participants in each group would have a similar mean and range of body mass index $\left(\mathrm{BMI} ; \mathrm{kg} / \mathrm{m}^{2}\right.$ ), and group differences would not be attributable to weight. Each control participant was recruited to be within one BMI point of an individual BED participant. Initial telephone screening assessed height, weight, and the above exclusionary criteria. Potential participants were interviewed using the EDE, administered the EAT and DASS, and their height and weight were measured by investigators. Control participants needed to score below 20 on the EAT and 15 on the DASS, and to have had no more than one binge episode or one episode of compensatory behavior (e.g., vomiting or laxative abuse) in the past month and no history of an eating disorder.

Approximately 150 women were screened by telephone as possible BED participants or weight-matched controls, 60 of them were interviewed, and 33 met inclusion criteria and were invited to participate. Three participants withdrew either due to scheduling conflicts or without giving a reason. The remaining $15 \mathrm{BED}$ and 15 control participants completed the study. Their mean age (SD) was 27.07 years (8.24), and mean BMI was 28.15 (6.27), with no age or BMI differences between groups. Sixty percent of participants in both the BED and control groups were overweight or obese (BMI $\geq 25$ ), and $40 \%$ were in the normal BMI range (20-25). The BED participants reported a mean of 23.20 (29.04) binge-eating episodes in the past 28 days.

\section{Procedures}

All procedures took place individually in a private room. Using a repeated-measures design, participants attended two test-meal sessions in counterbalanced order, conducted at the same time (between 11 a.m. and 3 p.m.) on two nonconsecutive days. Participants were instructed in advance to keep their eating and exercise patterns as similar as possible on both days of the study and not to eat or drink anything but water from $3 \mathrm{~h}$ prior 
to their sessions. They were also asked not to consume alcoholic beverages anytime after 5 p.m. on the evening before their sessions. Participants were telephoned the day before each session and reminded of these instructions. Before each test session, participants completed a brief diary of their food and beverage intake and exercise patterns from the previous $24 \mathrm{~h}$ to ensure that these instructions had been followed. Participants then completed a questionnaire assessing the severity of current physical symptoms (e.g., nausea, stomach upset, dizziness, and sleepiness) using a 10-point scale. Any participants endorsing symptoms (above five) were deemed unfit to participate and rescheduled.

Just before being served each preload drink, participants tasted a 15-ml sample removed from that day's preload and rated it for prospective consumption, estimated calorie content, creaminess, thickness, sweetness, and pleasantness (e.g., "How pleasant is the taste of this food right now?"), on 0-160 $\mathrm{mm}$ sample-rating scales ("visual analog scales;" VAS; ${ }^{25,26}$ ), anchored with the words the words Not at all/None and Extremely. Before and after consuming the drink, participants also rated their hunger, fullness, strength of desire to eat, prospective consumption, excitement about eating, satisfaction, desire to eat a meal, desire to eat a dessert, thirst, and nausea, on $0-160-\mathrm{mm}$ appetite rating scales. These scale questions were anchored with the words Not at all/None and Most imaginable. Participants were instructed to consume the contents of the drink over a $10-\mathrm{min}$ period and to keep track of the time using a provided clock so that they would be approximately halfway through at $5 \mathrm{~min}$. After $10 \mathrm{~min}$, the investigator returned and made sure the drink was completed. A buffet meal was served 30 min after initial presentation of the drink. Participants were not permitted to read or engage in other activities during the meal. Participants were instructed: "Relax and let yourself eat as much or as little as you feel like eating, taking as much or as little time as you wish. Allow yourself to eat whatever you feel like eating right now." They were instructed that, when they finished, they should knock on the door where they had initially met the investigator down the hall.

Before and after meals, participants completed the same appetite rating scales as before and after drinks, with two additional questions after meals on enjoyment of the meal and pleasantness of the food (e.g., "How pleasant have you found the food?"). After meals, additional questions assessed whether they had experienced a loss of control over eating: "Did you have a sense of loss of control at the time you were eating?" with response options including yes or no, in keeping with accepted assessment measures (e.g., Ref. 20) and the DSM-IV description of this criterion for binge-eating episodes. ${ }^{13}$ Participants were also asked how much they felt their recent eating episode had been typical of a meal, and how much it was typical of a binge, with responses on a five-point scale from Not at all typical to Extremely typical. They were also asked whether they felt they had eaten too much, with responses on a seven-point scale from Nothing at all to So much I can't go on.

\section{Preload Drinks}

Preloads (171 kcal, $18.1 \%$ protein, $55.6 \%$ carbohydrate, and $26.3 \%$ fat) were formulated with $250 \mathrm{ml}$ of Nature's Energy Chocolate Milk (Meadow Fresh NZ Ltd, Dunedin, New Zealand). The LV version consisted of the chilled milk alone, and the HV version consisted of the milk blended (with a hand-held blender) with $250 \mathrm{ml}$ water, five tablets of sucralose sweetener $(0.28 \mathrm{~g}$; Splenda, McNeil Nutritionals, LLC), 1.5 g carboxymethylcellulose thickener (CMC; Formula Foods) and $1 \mathrm{~g}$ unsweetened cocoa. Formulations were based on previous research using milk-based drinks to vary volume. ${ }^{2,3}$ The twofold difference between the drinks was based on previous studies that also used a twofold difference in volume of similar sizes (e.g., 200 vs. $400 \mathrm{ml}$ and 300 vs. $600 \mathrm{ml}$ ). ${ }^{2,4,12}$ After the development of the preloads, samples were rated by 10 university students (not study participants); no differences on $0-160 \mathrm{~mm}$ sample-rating scales were found between HV and LV drinks in prospective consumption, estimated calorie content, or enjoyment of taste or texture. Drinks were served chilled in an opaque, covered cup with a straw, to minimize cognitive and visual cues.

\section{Test Meals}

Buffet test meals served a variety of foods in ample amounts (Table 1). Test meals were accompanied by a 750 -ml bottle of spring water, serving utensils, and a plate. Meals were timed, and food was weighed on a digital scale to the nearest $0.1 \mathrm{~g}$ before and after meals, and the difference between pre- and postmeal weights was recorded as the amount eaten.

These procedures were approved by the Human Ethics Committee of the University of Canterbury. During debriefing, BED participants were given information on treatment options.

\section{Statistical Procedures}

Participants' hedonic responses and beliefs about energy content of the drink samples were compared using paired-sample $t$ tests. To test for main effects between LV and HV conditions and between BED and control participants, 2 (volume condition) $\times 2$ (participant type) factor ANOVAs, with repeated-measures on the first factor, were conducted on appetite ratings and food intake as dependent variables. BMI and EDE restraint subscale scores were tested as covariates in the analysis of food intake. Finally, chi-square analyses 
TABLE 1. Weight and nutritional composition of test-meal foods served

\begin{tabular}{|c|c|c|c|c|c|}
\hline Food & Amount served (g) & Energy (kJ/g) & PRO (\% of Energy) & CHO (\% of Energy) & Fat (\% of Energy) \\
\hline Chicken $^{\mathrm{a}}$ & 225 & 4.04 & 84.33 & 0.74 & 14.93 \\
\hline Bologna ${ }^{a}$ & 225 & 5.08 & 36.16 & 26.30 & 37.11 \\
\hline Chips & 200 & 23.00 & 4.50 & 38.70 & 57.86 \\
\hline Cheese & 225 & 13.70 & 21.09 & 4.27 & 75.40 \\
\hline Wheat bread & 170 & 10.25 & 13.03 & 74.46 & 7.72 \\
\hline White bread & 170 & 10.46 & 12.77 & 76.32 & 7.57 \\
\hline Tomato & 200 & 0.22 & 14.00 & 80.00 & 6.00 \\
\hline Cucumber & 130 & 0.13 & 20.00 & 80.00 & 0.00 \\
\hline Ice cream & 400 & 8.02 & 6.25 & 44.14 & 49.83 \\
\hline Cookies & 225 & 20.00 & 3.50 & 59.40 & 36.80 \\
\hline Apples & 470 & 0.60 & 1.00 & 94.00 & 5.00 \\
\hline Eggs $\mathrm{b}, \mathrm{c}$ & 200 & 5.96 & 35.87 & 0.84 & 63.89 \\
\hline Tuna ${ }^{b}$ & 200 & 4.59 & 90.23 & 1.56 & 8.21 \\
\hline Beans $^{c}$ & 275 & 4.95 & 23.28 & 58.7 & 6.85 \\
\hline
\end{tabular}

Nutritional details were derived from manufacturers' information and the U.S. Department of Agriculture National Nutrient Database Version 1.0, HealtheTech, Inc.

${ }^{a}$ Served to meateaters.

${ }^{\mathrm{b}}$ Served to nonmeat eaters.

'Served to nonmeat/nonfish eaters.

examined the frequency of reported loss of control over eating in the two conditions. Alpha level was set at 0.05 . Data were analyzed using SPSS, version 14.0 (Chicago, IL).

\section{Results}

\section{Scale Ratings}

Hedonic Ratings. Participants' prospective consumption, estimated calorie content, creaminess, thickness, or sweetness ratings did not differ between HV and LV samples. Rating of pleasantness was higher for the LV sample than the $\mathrm{HV}$ sample $[t(29)=3.57, p<.005]$.

Appetite Ratings. Before preload consumption, there were no differences between conditions for hunger, fullness, strength of desire to eat, prospective consumption, excitement about eating, satisfaction, desire to eat a meal, desire to eat a dessert, thirst, or nausea. Immediately following the intake of preloads, HV drinks led to significantly less hunger $[F(1,28)=7.74, p<.01$; Fig. 1], lower strength of desire to eat $[F(1,28)=7.46, p<.01]$, less desire to eat a dessert $[F(1,28)=4.81, p<.05]$, and marginally less excitement about eating $[F(1,37)=4.03, p$ $=.05]$ than LV drinks. Both before and after drinks, BED women were higher than controls on strength of desire to eat $[F(1,28)=7.50, p<.02 ; F(1,28)=$ $8.73, p<.01$, respectively], prospective consumption $[F(1,28)=9.15, p<0.01 ; F(1,28)=14.99, p<$ $.005]$, excitement about eating $[F(1,28)=5.08, p<$ $.05 ; F(1,28)=10.93, p<.005]$, and desire for a meal $[F(1,28)=6.17, p<.02 ; F(1,28)=8.13, p<.01]$ and dessert $[F(1,28)=12.45, p<.005 ; F(1,28)=9.87$, $p<.005]$. No interaction effects were found.
FIGURE 1. Ratings of hunger (a) and desire to eat (b) on visual analogue scales $(\mathrm{mm})$ across all participants in highvolume and low-volume preload conditions at four time points: prepreload, postpreload, premeal, and postmeal $\left({ }^{*} p<.01\right)$.
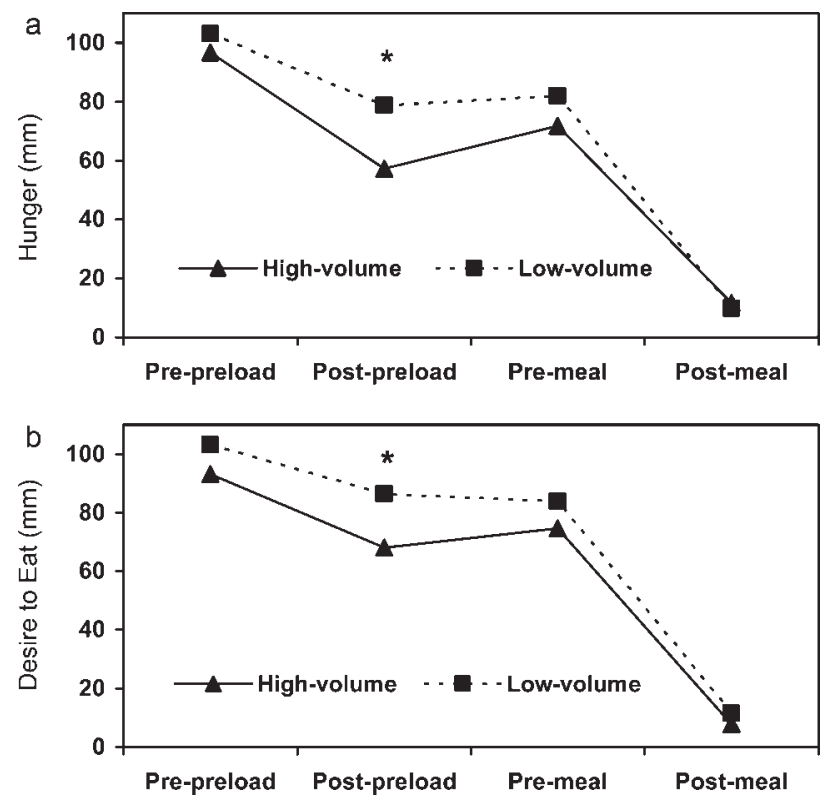

Twenty minutes after completion of preload drinks, just before test meals, excitement about eating was lower in the $\mathrm{HV}$ condition than in the $\mathrm{LV}$ condition $[F(1,28)=4.46, p<.05]$. BED women were higher than controls before meals on strength of desire to eat $[F(1,28)=6.46, p<.02]$, prospective consumption $[F(1,28)=10.58, p<.005]$, excitement about eating $[F(1,28)=9.98, p<.005]$, desire for a meal $[F(1,28)=10.04, p<.005]$ and desire for dessert $[F(1,28)=13.70, p<.005]$, and higher than controls after meals on nausea $[F(1,28)=5.05$, 
FIGURE 2. Mean test meal intake (SEM) after high-volume and low-volume preloads by BED participants and healthy weight-matched control participants.

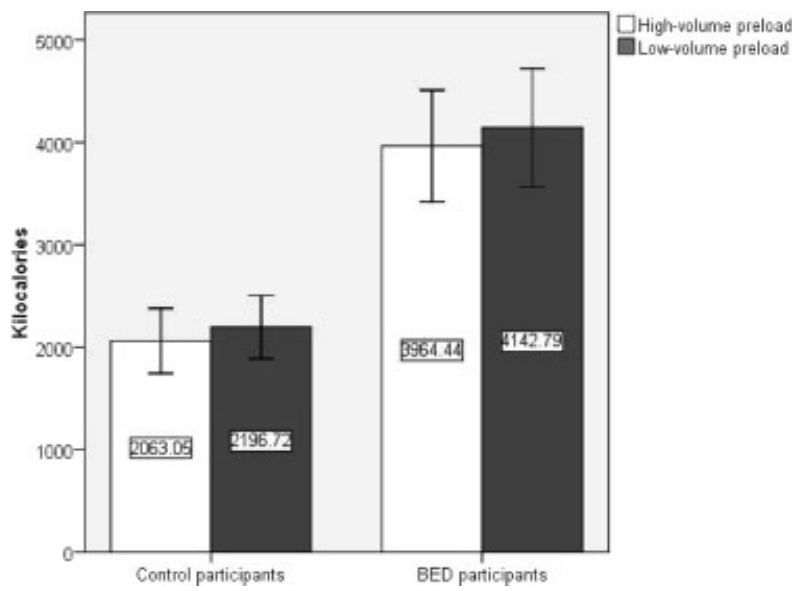

$p<.05]$. No other effects on appetite ratings were found after meals.

\section{Test Meals}

Food Intake. BED participants consumed more than control participants $[F(1,37)=11.10, p<.005$; Fig. 2]. Similar main effects for participant type were found for energy consumed from fat and from carbohydrates $[F(1,37)=10.88, p=.005 ; F(1,28)=$ $11.88, p<.005]$, but not for energy from protein or for grams of food consumed. Energy density of meals $(\mathrm{kcal} / \mathrm{g})$ was higher for BED than for controls $[F(1,37)=4.19, p<.05]$. BMI and restraint were not significant as covariates. No main effects of condition or interaction effects emerged.

Judgments about Food Intake. Across both conditions, BED participants were more likely to experience a loss of control over eating than non-BED participants (only one control reported a loss of control; $\left.\chi^{2}(1)=28.71, p<.001\right)$. In the HV condition, BED participants were equally likely to experience a loss of control $(53.3 \%)$ as not to experience one $\left[46.7 \% ; \chi^{2}(1)=0.07\right.$, n.s.], whereas in the LV condition, BED participants were significantly more likely to experience a loss of control $(86.7 \%)$ than not to experience one $\left[13.3 \% ; \chi^{2}(1)=8.07\right.$, $p<.005]$. Controls felt that the test meal was more typical of a meal than did BED participants $[F(1,28)$ $=17.92, p<.001]$, and BED participants felt more strongly than controls that they had eaten too much $[F(1,28)=7.88, p<.01]$. An interaction effect showed that BED participants felt that the test meal was more bingelike than control participants, but only in the HV condition $[F(1,28)=6.23$, $p<.05]$.

\section{Conclusion}

Women with BED and weight-matched control participants reported less hunger, desire to eat, desire for dessert, and excitement about eating after highvolume preloads than after low-volume preloads that were matched for hedonic properties. Preload volume did not influence subsequent food intake for either BED or control participants. However, preload volume did influence perceptions of loss of control during test meals in women with BED: after only LV drinks, the perception of loss of control was more frequent than the perception of control. Women with BED ate substantially more than controls at test meals, consistent with previous research showing that these individuals consume more food than controls throughout the day ${ }^{27}$ and during binge episodes. ${ }^{28}$ Women with BED also reported greater desire to eat, prospective consumption, excitement about eating, and desire for a meal and dessert than controls. These differences provide further evidence for the appetitive disturbances in BED.

These data suggest that increasing food volume may not be an effective method of curtailing binge eating and overeating in women with BED, but that it may have a psychological impact by decreasing perceived hunger and loss of control. It is possible that changing these perceptions might have therapeutic value in this population. A behavioral treatment designed primarily to change perceptions of hunger and fullness can effectively treat BED. ${ }^{29}$ Cognitive-behavioral therapy (CBT) systematically reintroduces patients to "forbidden foods" in a controlled manner, not because these foods are especially satiating or reduce subsequent intake, but because a goal of treatment is to reduce the frequency of loss of control over eating. ${ }^{30}$ The loss of control over eating, regardless of actual intake level, is closely related to eating disorder symptoms and general psychopathology. ${ }^{31}$

The present findings suggest that in the populations studied here, food volume might have an immediate effect on perceptions of satiation during or right after intake, but that this effect is shortlived. It is possible that the short-term effect of volume on appetite may be overridden by the powerful food environment and high individual 
susceptibility to that environment. ${ }^{32}$ Both of the groups studied here, frequent binge eaters and healthy but primarily $(60 \%)$ overweight BMImatched controls, may be especially vulnerable to the "toxic food environment,"33 recreated in some respects by the buffet-style meal offering multiple foods in ample amounts. Indeed, average food intake was quite large overall. These results contradict past studies demonstrating an effect of volume on food intake. ${ }^{2,4-6}$ However, they are consistent with three studies with lean, healthy participants showing that increasing the volume of foods enhanced satiety ratings but did not influence actual food intake, ${ }^{10,12}$ even when test meals followed the independent variables immediately with no delay. ${ }^{11}$ It has been proposed that gastric volumetric signals limit meal size through negative feedback on only short-term ingestion. ${ }^{34}$

Other authors attempting to explain different findings across studies have suggested that the choice of soup versus milk-based preloads, food volume or energy content, test meal ingredients, or single-item versus multiple-item test meals may account for varying results. ${ }^{10,12}$ This study used milk-based drinks and multiple-item test meals, similar to the studies of Rolls et al., which found volume effects on food intake. However, the energy content of preloads in this study (715 kJ) was closer to those of Norton and colleagues $(1121 \mathrm{~kJ})$ or Gray and colleagues $(629 \mathrm{~kJ})$ than to the relatively higher energy drinks of Rolls and colleagues (2088 kJ), suggesting that volume may be more influential when intake is large, as proposed by Gray et al. ${ }^{10}$ This study's drink energy level was determined based on the potential therapeutic application of findings; when patients who are overweight are asked to ingest regular snacks between meals (as prescribed in $\left.\mathrm{CBT} ;{ }^{30}\right)$, solid or liquid snacks need to be moderately sized. It is also possible that a greater than twofold difference in volume between the drinks (were it feasible to hold hedonic factors constant) might have produced differential food intake. However, the absence of even a trend for food intake differences between conditions suggests that a larger difference in volume between conditions might still not have produced a significant effect. It is possible that even at greater energy levels and volume differences across conditions, energy content might be the key determinant of subsequent food intake, particularly over time.

Another difference between the current and prior research is the removal of visual cues. Although volume may affect food intake even when visual and sensory cues are bypassed, ${ }^{4}$ past studies have aimed to maximize visual and cognitive cues related to volume by serving drinks in a clear uncovered glass (e.g., Ref. 3). In contrast, this study aimed to eliminate the potential confound of visual or cognitive cues and to isolate orosensory and gastric effects by serving drinks in opaque covered cups with a straw. The effect of volume on food intake found in some previous studies may have been partly cognitive in nature, and this mechanism may have been reduced in the present study. Research on the influence of portion size attests to the powerful role of cognitive cues in determining food intake. ${ }^{35,36}$ Finally, it is even possible that cultural differences might have contributed to the differences across studies; research finding no effect of volume on food intake was conducted in the United Kingdom, while research finding the effect was conducted in the United States. The New Zealand sample of the present investigation would much more closely resemble U.K. participants in their eating-related customs and patterns. However, the mechanism for such cultural differences is unknown.

The BED participants' greater food intake, loss of control, feeling of having eaten too much, and desire and excitement ratings than controls provide additional evidence for the validity and utility of the laboratory test meal and appetite rating scales as tools for behaviorally assessing binge eating and appetite in BED. ${ }^{37}$ These differences are consistent with the current diagnostic criteria for binge eating: eating a large amount of food in a discrete period of time while experiencing a loss of control over eating. ${ }^{13}$ The higher energy density of meals in BED is also consistent with previous findings that binges are larger than those of weight-matched controls $^{18,38}$ and often consist of foods high in energy density. ${ }^{39-41}$ Finally, the increased nausea among BED participants after eating is consistent with the BED diagnostic criteria of feeling disgusted or uncomfortably full after binge eating.

A limitation of this study was the difference in pleasantness perceptions between $\mathrm{HV}$ and $\mathrm{LV}$ drinks. This difference was also found in previous studies. ${ }^{10,12}$ However, it did not contribute to food intake, as intake did not differ between conditions, and pleasantness ratings did not correlate with test meal intake in either condition. The volume of food consumed at meals (e.g., the amount of threedimensional space occupied by foods) was not measured, so that it was not possible to assess the combined volume of dependent and independent variables (milks plus meals). In addition, although this study held energy constant across different levels of volume, another way to measure the effects of volume independent of energy is to hold volume constant across different levels of energy. Future 
research could use this technique in women with BED. Future studies might also investigate the effects of volume alone, in the absence of energy content, rather than consumed in an energy-containing food to examine the effects of volume independent of food. It would also be interesting to apply a research design used in several previous studies, where participants are given instructions to binge or not to binge; these instructions effectively manipulate BED participants' mindset and behavior in laboratory-feeding studies (e.g., Ref. 18). The effects of volume on food intake might differ according to the mindset of the individual consuming the meal. Finally, future research is needed to investigate additional ways to improve satiety and reduce binge eating in individuals with BED. In light of the increasing prevalence of obesity and binge eating in the current toxic food environment, specifying the properties of food and the environment that can prevent overeating is imperative for these vulnerable individuals.

The authors acknowledge the contributions of $\mathrm{Mr}$. David Rout (Formula Foods) and Dr. Courtney Clyne.

\section{References}

1. Hedley AA, Ogden CL, Johnson CL, Carroll MD, Curtin LR, Flegal KM. Prevalence of overweight and obesity among US children, adolescents, and adults 1999-2002. JAMA 2004;291:2847-2850.

2. Rolls BJ, Castellanos VH, Halford JC, Kilara A, Panyam D, Pelkman $\mathrm{CL}$, et al. Volume of food consumed affects satiety in men. Am J Clin Nutr 1998;67:1170-1177.

3. Bell EA, Roe LS, Rolls BH. Sensory-specific satiety is affected more by volume than by energy content of a liquid food. Physiol Behav 2003;78:593-600.

4. Rolls BJ, Roe LS. Effect of the volume of liquid food infused intragastrically on satiety in women. Physiol Behav 2002;76: 623-631.

5. Rolls BJ, Bell EA, Thorwart ML. Water incorporated into a food but not served with a food decreases energy intake in lean women. Am J Clin Nutr 1999; 70:448-455.

6. Rolls BJ, Bell EA, Waugh BA. Increasing the volume of a food by incorporating air affects satiety in men. Am J Clin Nutr 2000; 72:1-8.

7. Bell EA, Castellanos VH, Pelkman CL, Thorwart ML, Rolls BJ. Energy density of foods affects energy intake in normal-weight women. Am J Clin Nutr 1998;67:412-420.

8. Bell EA, Rolls BJ. Energy density of foods affects energy intake across multiple levels of fat content in lean and obese women. Am J Clin Nutr 2001;73:1010-1018.

9. Stubbs RJ, Johnstone AM, Harbron CG, Reid C. Covert manipulation of energy density of high carbohydrate diets in 'pseudo free-living' humans. Int J Obes Relat Metab Disord 1998;22: 885-892.

10. Gray RW, French SJ, Robinson TM, Yeomans MR. Dissociation of the effects of preload volume and energy content on subjective appetite and food intake. Physiol Behav 2002;76:57-64.
11. Gray RE, French SJ, Robinson TM, Yeomans MR. Increasing preload volume with water reduces rated appetite but not food intake in healthy men even with minimum delay between preload and test meal. Nutr Neurosci 2003;6:29-37.

12. Norton GN, Anderson AS, Hetherington MM. Volume and variety: Relative effects on food intake. Physiol Behav 2006;87: 714-722.

13. American Psychiatric Association. Diagnostic and Statistical Manual of Mental Disorders, 4th ed. Washington, DC: American Psychiatric Press Text Revision, 2000.

14. Latner JD, Wilson GT. Self-monitoring and the assessment of binge eating. Behav Ther 2002;33:465-477.

15. Geliebter A, Hashim SA. Gastric capacity in normal, obese, and bulimic women. Physiol Behav 2001;74:743-746.

16. Geliebter A, Melton PM, McCray RS, Gallagher DR, Gage D, Hashim SA. Gastric capacity, gastric emptying, and test-meal intake in normal and bulimic women. Am J Clin Nutr 1992;56: 656-661.

17. Geliebter A, Yahav EK, Gluck ME, Hashim SA. Gastric capacity, test meal intake, and appetitive hormones in binge eating disorder. Physiol Behav 2004;81:735-740.

18. Guss JL, Kissileff HR, Zimmerli E, Walsh BT, Devlin MJ. Binge size increases with body mass index in subjects with binge eating disorder. Obes Res 2002;10:1021-1029.

19. Geliebter A. Gastric distension and gastric capacity in relation to food intake in humans. Physiol Behav 1988;44:665-668.

20. Fairburn CG, Cooper Z. The eating disorder examination. In: Fairburn CG, Wilson GT, editors. Binge Eating: Nature, Assessment, and Treatment, 12th ed. New York: Guilford Press, 1993, pp. 317-332.

21. Rivzi SL, Peterson CB, Crown SJ, Agras WS. Test-retest reliability of the eating disorder examination. Int J Eat Disord 2000;28: 313-316.

22. Garner DM, Garfinkel PE. The eating attitudes test: An index of the symptoms of anorexia nervosa. Psychol Med 1979;9:273279 .

23. Lovibond PF, Lovibond SH. The structure of negative emotional states: Comparison of the Depression Anxiety Stress Scales (DASS) with the Beck depression and anxiety inventories. Behav Res Ther 1995;33:335-343.

24. American Psychiatric Association. Diagnostic and Statistical Manual of Mental Disorders, 4th ed. Washington, DC: American Psychiatric Press, 1994.

25. Flint A, Raben A, Blundell JE, Astrup A. Reproducibility, power and validity of visual analogue scales in assessment of appetite sensations in single test meal studies. Int J Obes Relat Metab Disord 2000;24:38-48.

26. Stubbs RJ, Hughes DA, Johnstone AM, Rowley E, Reid C, Elia M, et al. The use of visual analogue scales to assess motivation to eat in human subjects: A review of their reliability and validity with an evaluation of new hand-held computerized systems for temporal tracking of appetite ratings. Br J Nutr 2000;84:405415 .

27. Rossiter EM, Agras WS, Telch CF, Bruce B. The eating patterns of non-purging bulimic subjects. Int J Eat Disord 1992;11:111-120.

28. Yanovski SZ, Leet M, Yanovski JA, Flood M, Gold PW, Kissileff HR, et al. Food selection and intake of obese women with and without binge eating disorders. Am J Clin Nutr 1992;56:979-980.

29. Craighead LW, Allen HN. Appetite awareness training: A cognitive behavioral intervention for binge eating. Cogn Behav Pract 1995;2:249-270.

30. Fairburn CG, Marcus MD, Wilson GT. Cognitive-behavioral therapy for binge eating and bulimia nervosa: A comprehensive manual. In: Fairburn CG, Wilson GT, editors. Binge Eating: Nature, Assessment, and Treatment. New York: Guilford Press, 1993, pp. 361-404. 
31. Latner JD, Hildebrandt T, Rosewall JK, Chisholm AM, Hayashi $\mathrm{K}$. Loss of control over eating reflects eating disturbances and general psychopathology. Behav Res Ther 2007;45:22032211.

32. Lowe MR, Levine AS. Eating motives and the controversy over dieting: Eating less than needed versus less than wanted. Obes Res 2005;13:797-806.

33. Brownell KD, Horgen KB. Food Fight: The Inside Story of the Food Industry, America's Obesity Crisis, and What We Can Do About It. New York, NY: McGraw Hill, 2004.

34. Powley TL, Phillips RJ. Gastric satiation is volumetric, intestinal satiation is nutritive. Physiol Behav 2004;82:6974.

35. Ello-Martin JA, Ledikwe JH, Rolls BH. The influence of food portion size and energy density on energy intake: Implications for weight management. Am J Clin Nutr 2005;82(Suppl):2365241S.
36. Diliberti N, Bordi PL, Conklin MT, Roe LS, Rolls BJ. Increased portion size leads to increased energy intake in a restaurant meal. Obes Res 2004;12:562-568.

37. Anderson DA, Williamson DA, Johnson WG, Grieve CO. Validity of test meals for determining binge eating. Eat Behav 2001;2: 105-112.

38. Raymond NC, Bartholome LT, Lee SS, Peterson RE, Raatz SK. A comparison of energy intake and food selection during laboratory binge eating episodes in obese women with and without a binge eating disorder diagnosis. Int J Eat Disord 2007;40:67-71.

39. Alvarenga MS, Negrao AB, Phillippi ST. Nutritional aspects of eating episodes followed by vomiting in Brazilian patients with bulimia nervosa. Eat Weight Disord 2003;8:150-156.

40. Kales EF. Macronutrient analysis of binge eating in bulimia. Physiol Behav 1990;48:837-840.

41. Drewnowski A. Taste responsiveness in eating disorders. Ann NY Acad Sci 1989;575:399-409. 